\section{Spotted Substrates for Focal Presentation of Proteins to Cells}

Biotechniques 33:1018-1024 (November 2002)

\begin{abstract}
Neurites and other cell processes encounter localized deposits of signaling factors as they grow. The difficulty in generating patterned artificial substrates has hindered the analysis of these instructive factors in vitro. Here we report a simple method for presenting cultured cells with small spots of protein on an otherwise uniform substrate. We use a biolistic device called a gene gun to deposit 0.1-5 $4 \mathrm{~m}$ fluorescent dots of pure protein on or beneath a growth-promoting substrate. Using this technique, we demonstrate local presynaptic differentiation of motoneurons in response to dots of a polycation. We also show that biotin-avidin and antibody-antigen interactions can be used to prepare spots from more dilute, more labile, or less abundant proteins. This method should prove useful for analyzing extracellular signaling molecules that act focally on neurons or other cell types.
\end{abstract}

\section{INTRODUCTION}

Neuronal processes (axons and dendrites) grow, turn, branch, stop, and form synapses in response to highly localized signals in their environment (12). Although this subcellular specialization is especially prominent in neurons, many cell types make local responses to local cues as they differentiate, become polarized, or migrate.

To understand the mechanisms that underlie such local phenomena, it is obviously important to present putative cues to restricted portions of the cell. However, in many cases, cell-environment interactions have been analyzed in vitro by growing the cells on substrata uniformly coated with putative signaling molecules or by applying molecules in soluble form. In large part, this is because methods available for constructing patterned substrates involve laborious, complex, or expensive tech- niques such as photolithography, microstamping, or microfluidic patterning $(2,3,6,7,13)$. Here we report a simple, alternative approach in which small dots of protein are applied to culture dishes ballistically (or biolistically) using a gene gun (8). In this system, the cells encounter spots of protein immobilized on an otherwise uniform substrate. We demonstrate the utility of the method by showing that neurites of primary motoneurons form varicosities and cluster synaptic vesicles at points of apposition to a known inducer of presynaptic differentiation (5). We also provide protocols for exploiting biotinavidin and antibody-antigen interactions to prepare spots with proteins that are dilute, labile, or in short supply.

\section{MATERIALS AND METHODS}

\section{Making Dots}

Eight-well chamber slides (Nalge Nunc International, Rochester, NY, USA) were coated with polyornithine $(1.5 \mu \mathrm{g} / \mathrm{mL}$; Sigma, St. Louis, MO, USA) in sterile water for $30 \mathrm{~min}$ and dried. The slides were then positioned so that the opening of a single well was centered on, and directly opposed to, a tissue culture membrane insert attached to a Helios gene gun (Bio-Rad Laboratories, Hercules, CA, USA) (Figure 1A). Neighboring wells were masked with Parafilm ${ }^{\circledR}$. The gun was fired as described below, the chamber slide and the Parafilm were repositioned, and the process was repeated until all wells had been spotted. Slides were left to dry under sterile conditions for $30 \mathrm{~min}$ and then were coated with a solution of mouse laminin $(10 \mu \mathrm{g} / \mathrm{mL}$; Invitrogen, Carlsbad, CA, USA) in L-15 medium at $37^{\circ} \mathrm{C}$ overnight.

To prepare cartridges, Gold-Coat tubing (Bio-Rad Laboratories) was coated with $1 \%$ polyvinylpyrrolidone in ethanol, as recommended by the manufacturer, and then cut into 1-1.5 in lengths. The tubing was syringeloaded with proteins dissolved in sterile PBS and left for 5 min before being drained, cut with the Tubing Cutter (Bio-Rad Laboratories) into 0.5-in segments, and loaded into a Helios gene gun. Delays greater than $10 \mathrm{~min}$ be- tween draining and firing a cartridge greatly reduced the amount of protein reaching the dish surface, probably because of drying within the tube.

To adjust the dot size, a $25-\mathrm{mm}$ sterile polycarbonate tissue culture insert (Nalge Nunc International) of either 3or $8-\mu \mathrm{m}$ pore size was fitted over the barrel liner of the gene gun and secured with tape (Figure 1A). To accommodate the filter, we removed the spacers from the end of the barrel liner. Helium pressure was adjusted to 80 or 120 psi for the 3- or 8- $\mu \mathrm{m}$ membrane, respectively. Before shooting a new protein solution, the membranes were replaced or cleared with several blank shots to eliminate cross-contamination.

The sources and concentrations of proteins applied biolistically are as follows: BSA $(3.3 \mathrm{mg} / \mathrm{mL}$ for motorneuron culture; otherwise $200 \mu \mathrm{g} / \mathrm{mL}$ ) (Sigma); $200 \mu \mathrm{g} / \mathrm{mL}$ BSA conjugated to Alexa Fluor (Ax) 488 (BSA-Ax488) (Molecular Probes, Eugene, OR, USA); $1.5 \mathrm{mg} / \mathrm{mL}$ poly-D-lysine; $2 \mathrm{mg} /$ $\mathrm{mL}$ fluorescein isothiocyanate (FITC)conjugated poly-D-lysine (fPL) (both from Sigma); $200 \mu \mathrm{g} / \mathrm{mL}$ biotin-conjugated $\mathrm{BSA}$; and $200 \mu \mathrm{g} / \mathrm{mL}$ donkey anti-goat IgG Ax 488 conjugate (Molecular Probes).

To generate multilayered dots, the slides were shot as above, washed three times with PBS, and then incubated with one of the following proteins diluted in L-15 medium supplemented with FCS or BSA: $25 \mu \mathrm{g} / \mathrm{mL}$ rhodamine-conjugated avidin, $10 \mu \mathrm{g} / \mathrm{mL}$ streptavidin-Ax 568, $5 \mu \mathrm{g} / \mathrm{mL}$ biotinconjugated goat anti-mouse $\operatorname{IgG}$ (Amersham Biosciences, Piscataway, NJ, USA), $2 \mu \mathrm{g} / \mathrm{mL}$ goat anti-mouse IgG2a Ax 568 conjugate, donkey antigoat IgG Ax 568 conjugate, or donkey anti-goat IgG Ax 488 conjugate.

\section{Tissue Culture}

Motoneurons were purified from E12.5 mouse embryos by density centrifugation at $500 \times g$ for $15 \mathrm{~min}$ in OptiPrep (Sigma) as previously described (1). Purified motoneurons were plated onto spotted, laminin-coated chamber slides and grown for five days in Neurobasal media (Invitrogen) with B27 supplement (2\% v/v) (Invitrogen), horse serum $(2 \% \mathrm{v} / \mathrm{v}), 0.5 \mathrm{mM}$ L-glutamine, 
$25 \mu \mathrm{M}$ L-glutamate, $25 \mu \mathrm{M} \beta$-mercaptoethanol, and $10 \mathrm{ng} / \mathrm{mL} \mathrm{BDNF}$ at $37^{\circ} \mathrm{C}$ and $7.5 \% \mathrm{CO}_{2}$.

\section{Histology}

Motoneurons were fixed with $4 \%$ paraformaldehyde in PBS for $15 \mathrm{~min}$ at room temperature. The cells were washed three times with PBS before the remaining paraformaldehyde was scavenged, and the cells were permeabilized with $50 \mathrm{mM}$ lysine in PBS plus Triton ${ }^{\circledR}$ $\mathrm{X}-100(0.1 \% \mathrm{v} / \mathrm{v})$ for $15 \mathrm{~min}$. Nonspecific staining was blocked with a solution of BSA $(2 \% \mathrm{v} / \mathrm{v})$ and goat serum $(2 \% \mathrm{v} / \mathrm{v})$ plus Triton X-100 (0.1\% v/v) in PBS for $1 \mathrm{~h}$ at room temperature. The cells were then incubated with a mouse monoclonal anti-synaptic vesicle protein-2 (SV2) antibody (Developmental Studies Hybridoma Bank, Iowa City, IA, USA) plus anti-neurofilament-200 rabbit polyclonal antibody (Sigma) overnight at $4^{\circ} \mathrm{C}$. The cells were washed with PBS, incubated with indocarbocyanine (Cy3)-conjugated goat antimouse IgG (Jackson ImmunoResearch, West Grove, PA, USA) plus goat antirabbit $\mathrm{IgG} A x 350$ conjugate for $1 \mathrm{~h}$ at room temperature, washed again with PBS, and mounted with glycerol plus paraphenylenediamine.

Images were collected on a Axioplan2 microscope (Carl Zeiss, Thornwood, NY, USA) with a MagnaFire camera (Optronics, Goleta, CA, USA) and rendered with Adobe ${ }^{\circledR}$ Photoshop ${ }^{\circledR}$ version 6.0.

\section{RESULTS AND DISCUSSION}

The gene gun uses a burst of pressurized helium to propel the particles. Although it is designed to shoot DNAcoated gold microspheres, we found that it is also able to spray solutions onto culture dishes similar to an atomizer. In initial tests, the spray pattern consisted of large, irregular splotches, whereas we wanted to present the neurites with dots approximately $1 \mu \mathrm{m}$ in diameter. Therefore, we affixed a porous polycarbonate tissue culture insert over the barrel of the gene gun to break up the droplets (Figure 1A). With this modification, smaller dots were produced over an area that was approximately $4 \mathrm{~mm}$ in diameter. The dot size varied with the pore size of the membrane affixed to the gun: a $3-\mu \mathrm{m}$ pore size generated dots with diameters of $0.1-1 \mu \mathrm{m}$, while an $8-\mu \mathrm{m}$ pore size generated dots that ranged up to $5 \mu \mathrm{m}$ in diameter (Figure 1, B-D).

\section{Local Responses of Motor Neurites to Dots}

Neurites growing on large (approximately $50 \mu \mathrm{m}$ ) beads coated with poly- 


\section{Short Technical Reports}

D-lysine show multiple sites of presynaptic differentiation in which synaptic vesicles are clustered within swellings of neurites (5). To examine the utility of our method, we prepared a dish spotted with polylysine using an $8-\mu \mathrm{m}$ membrane. The unmodified polylysine was mixed with fluorescein-conjugated polylysine so that the dots would be identifiable. Other wells were spotted with a mixture of BSA and BSAAx488 as a control. Laminin was then applied to all wells, and embryonic motorneurons were plated in them.
The frequency and intensity of polylysine- or BSA-containing dots in cultures maintained for five days did not differ substantially from those examined immediately after spotting, thus demonstrating the long-term stability of spotted substrates. Neurites accumulated SV2 at points of contact with polylysine dots (Figure 2, A-D), indicating the presence of synaptic vesicles $(4,11)$. In addition, the neurite diameter often increased locally at the dots, forming varicosities similar to those observed at developing neuromuscular junctions in vivo (10).
These results demonstrate both molecular and morphological presynaptic differentiation. Neurites that encountered BSA dots did not accumulate SV2 or form varicosities (Figure 2, E-H). These results show that spotted substrates can elicit biologically meaningful localized responses to patterned signals.

\section{Using Dots for Protein Capture}

Spotting requires a relatively large quantity of protein (approximately 100 $\mu \mathrm{L}$ protein solution at an active concen-
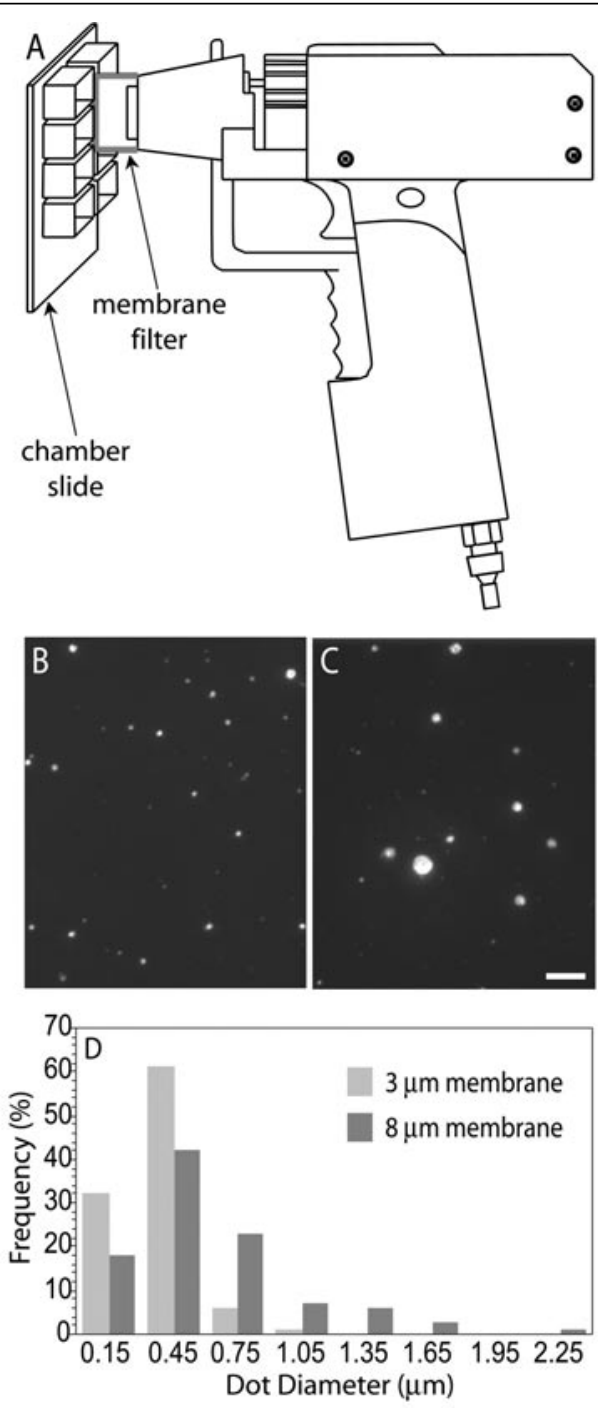

Figure 1. Biolistic generation of spotted substrates. Chamber slides were held against the membrane of a tissue culture insert affixed over the gene gun detachable barrel (A). Parafilm was used to mask the neighboring wells from cross contamination. A solution of BSA-Ax488 was shot through tissue culture filter inserts with 3 (B) or $8 \mu \mathrm{m}$ (C) pores at 80 or $120 \mathrm{psi}$, respectively. (D) The distribution of the dot sizes depends on the membrane pore size. For panels $B$ and $\mathrm{C}$, scale bar equals $5 \mu \mathrm{m}$.

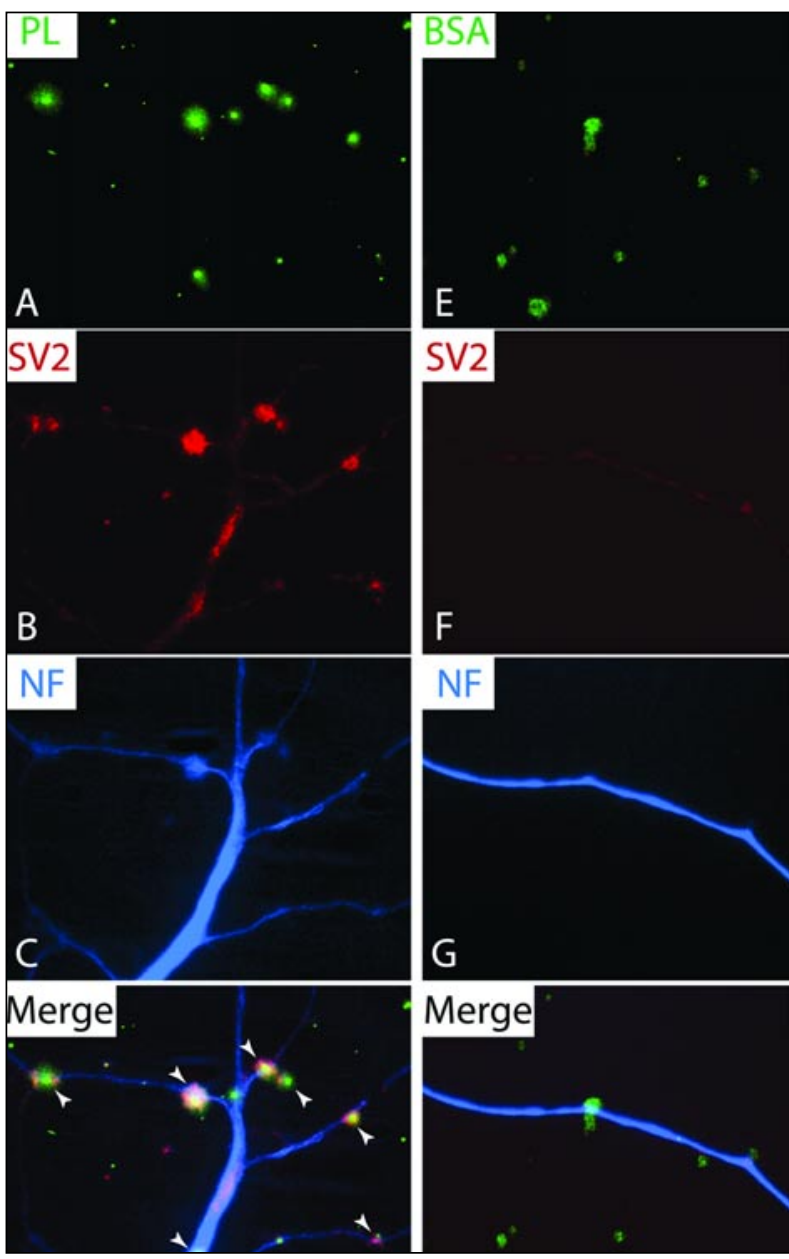

Figure 2. Polylysine dots stimulate the local clustering of SV2 protein and formation of synaptic varicosities. Embryonic mouse motoneurons were cultured on laminin-coated microchambers pre-spotted with either polylysine (PL) supplemented with fluorescein-PL (green, A) or BSA supplemented with BSA488 (green, B). After five days cells were fixed and stained for SV2 (red, B and F) and neurofilament-200 (blue, C and G). Digital images were collected and combined demonstrating colocalization of SV2 and varicosities over PL dots (D) but not over control BSA dots $(\mathrm{H})$. Scale bar $(\mathrm{A}-\mathrm{H})$ equals $5 \mu \mathrm{m}$. 


\section{Short Technical Reports}

tration) with an activity that is stable to drying on the dish. Polylysine is well suited for spotting because it is readily available, pure, inexpensive, and stable. However, few proteins capable of eliciting biological responses are likely to have these qualities. To circumvent these limitations, we tested the possibility of using biotin-avidin and antibody-antigen interactions to trap the proteins on the spots.

To assess the ability of biotin to recruit avidin to the dots, polyornithinecoated chamber slides were spotted with biotinylated BSA plus BSAAx488, coated with laminin, and then incubated with rhodaminated avidin (in L-15 medium supplemented with BSA for $12 \mathrm{~h}$ at room temperature). After washing, avidin was specifically bound to the dots (Figure 3A). Identical dishes spotted with BSA plus BSA-Ax488 before incubation with rhodamineavidin showed no avidin binding (Figure 3B).

To assess the ability of immobilized avidin to bind a biotinylated protein to the dots, we attempted to spot dishes with avidin. However, we found that avidin immobilized in this way was unable to bind significant quantities of biotinylated protein (data not shown), perhaps because of inactivation during drying (9). To overcome this problem, the slides were spotted with biotinylated BSA and then coated with laminin and treated sequentially with streptavidin-Ax568 and biotinylated goat antimouse IgG. To detect bound biotinylated protein, we added donkey anti-goat Ax488. Following this sequence, streptavidin and biotinylated goat antimouse were both specifically bound to the dots (Figure 3C). In contrast, when biotinylated goat anti-mouse was omitted from the second incubation, no binding of donkey anti-goat Ax488 was detected (Figure 3D). This method of linking proteins to the dish surface should prove useful if a putative signaling factor can be purified and biotinylated. The high concentration of biotinylated BSA spotted on the dish, plus the multivalency of avidin, ensures that any biotinylated protein added subsequently will become highly concentrated on the dots.

If antibodies (unlike avidin) retained their binding activity after spotting, then antibody-spotted dishes would allow concentration of putative signaling factors, even from impure preparations such as medium conditioned by transfected cells. To test this possibility, we spotted slides with donkey anti-goat IgG Ax488 plus BSA-Ax488. After laminin coating, the slides were incubated with goat IgG Ax568 (goat antimouse in L-15 medium supplemented with FBS) for $12 \mathrm{~h}$ at room temperature. Upon examination, goat IgG was found specifically bound to the dots (Figure 3E). Identical dishes incubated with donkey IgG Ax-568 after spotting instead of goat IgG Ax568 showed no binding (Figure 3F).

\section{CONCLUSIONS}

Patterned substrates represent an important step in the progression of in vitro assay systems toward the ultimate goal of replicating in vivo environments. Assays involving such substrates will become increasingly valuable as more localized cues are discovered. In some cases, specific patterns (e.g., stripes) may be needed, and sophisticat-

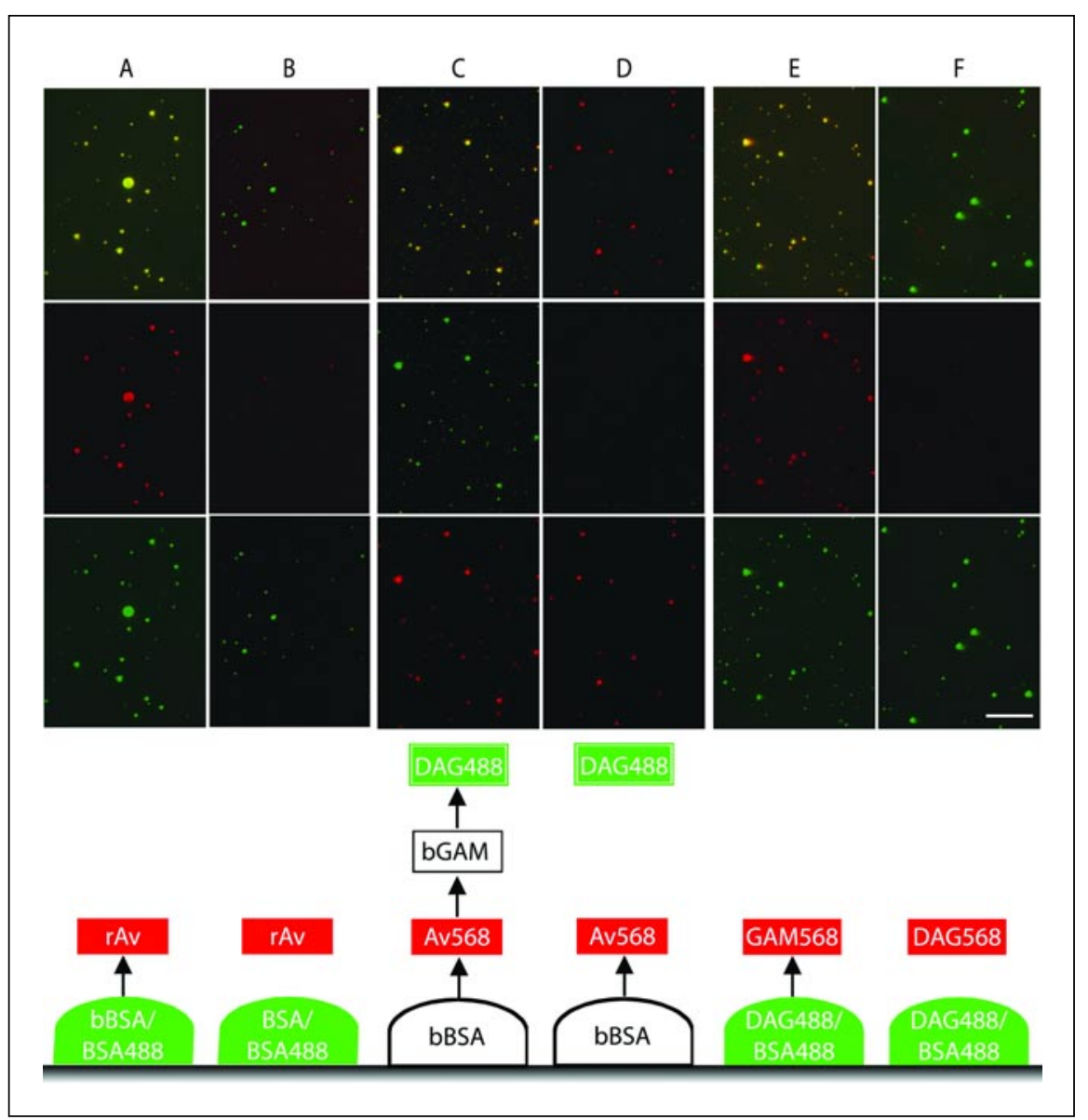

Figure 3. Use of biotin or antibody-containing spots to concentrate proteins. (A-F) Schematics under each image column depict the sequence of the binding steps. For each column, the lowest image corresponds to the first (lowest) fluorescent treatment in the schematic below. The middle image represents the next fluorescent treatment, and the upper image shows an overlay of the lower two images. (A) When dishes spotted with biotinylated BSA plus BSA-Ax488 are incubated with rhodaminated avidin, avidin is bound to the dots with a high specificity. (B) If nonbiotinylated BSA plus BSA-Ax488 are shot instead, the avidin is not bound. (C) Red dots are observed when the dishes are spotted with only biotinylated BSA and incubated with streptavidin-Ax568. When such dots are incubated with biotinylated goat anti-mouse $\mathrm{IgG}$, antibody is bound to the dots, as detected with donkey anti-goat Ax488. (D) When the biotinylated antibody is excluded, bound streptavidin cannot form this link. Antibodies can also form selectively adhesive dots. (E) Donkey anti-goat Ax488 plus BSA-Ax488 spots are able to bind goat anti-mouse Ax568 antibodies with high specificity. (F) These dots do not bind the non-complementary donkey anti-goat Ax568 antibody. Av, avidin; DAG, donkey anti-goat Ig; GAM, goat anti-mouse Ig. Scale bar equals $10 \mu \mathrm{m}$. 
ed methods for generating them are being developed in many laboratories. However, in many cases, the geometric requirements are not stringent, and technical simplicity and speed are more desirable. Spotted substrates should prove useful in such cases.

We were also able to develop techniques to couple proteins to dots formed from selectively adhesive materials. These modifications allow proteins that are less abundant, pure, or stable to be used in a spotted dish assay, thereby expanding the range of signals that can be tested. Together, these methods should be applicable to assays of a variety of putative cues and the analysis of their effects on diverse cell types.

\section{REFERENCES}

1.Arce, V., A. Garces, B. de Bovis, P. Filippi, C. Henderson, B. Pettmann, and O. deLapeyriere. 1999. Cardiotrophin-1 requires LIFR $\beta$ to promote survival of mouse motoneurons purified by a novel technique. J. Neurosci. Res. 55:119-126.

2.Bernard, A., E. Delamarche, H. Schmid, B. Michel, H.R. Bosshard, and H. Biebuyck. 1998. Printing patterns of proteins. Langmuir 14:2225-2229.

3.Blawas, A.S. and W.M. Reichert. 1998. Protein patterning. Biomaterials 19:595-609.

4.Buckley, K. and R.B. Kelly. 1985. Identification of a transmembrane glycoprotein specific for secretory vesicles of neural and endocrine cells. J. Cell Biol. 100:1284-1294.

5.Burry, R.W. 1980. Formation of apparent presynaptic elements in response to poly-basic compounds. Brain Res. 184:85-98.

6.Delamarch, E., A. Bernard, H. Schmid, A. Bietsch, B. Michel, and H. Biebuyck. 1998. Microfluidic networks for chemical patterning of substrates: design and application to bioassays. J. Am. Chem. Soc. 120:500-508.

7.Folch, A. and M. Toner. 2000. Microengineering of cellular interactions. Annu. Rev. Biomed. Eng. 2:227-256.

8.Johnston, S.A. and D.C. Tang. 1994. Gene gun transfection of animal cells and genetic immunization. Methods Cell Biol. 43PtA:353-365.

9.Reznik, G.O., S. Vajda, C.R. Cantor, and T. Sano. 2001. A streptavidin mutant useful for directed immobilization on solid surfaces. Bioconjug. Chem. 12:1000-1004.

10.Sanes, J.R. and J.W. Lichtman. 2001. Induction, assembly, maturation and maintenance of a postsynaptic apparatus. Nat. Rev. Neurosci. 2:791-805

11.Scranton, T.W., M. Iwata, and S.S. Carlson. 1993. The SV2 protein of synaptic vesicles is a keratan sulfate proteoglycan. J. Neurochem. 61:29-44.

12.Tessier-Lavigne, M. and C.S. Goodman. 1996. The molecular biology of axon guidance. Science 274:1123-1133.

13.Walter, J., B. Kern-Veits, J. Huf, B. Stolze, and F. Bonhoeffer. 1987. Recognition of position-specific properties of tectal cell membranes by retinal axons in vitro. Development 101:686-696.

We thank Dennis Oakley for help and Steve Carlson for valuable discussions. This work was supported by grants from the $\mathrm{Na}$ tional Institutes of Health (J.R.S. and R.O.L.W.) and the Medical Scientist Training Program grant no. T32 GM07200-28 to T.T.K. Address correspondence to Dr. Joshua R. Sanes, Washington University School of Medicine, 660 South Euclid, Campus Box 8108, St. Louis, MO 63110, USA.e-mail: sanesj@pcg.wustl.edu

Received 4 June 2002; accepted 3 July 2002.

Terrance T. Kummer, Hiroshi
Nishimune, Rachel O.L.
Wong, and Joshua R. Sanes
Washington University
School of Medicine
St. Louis, MO, USA

\section{Automated Colorimetric Screen for Apyrase Inhibitors}

BioTechniques 33:1024-1030 (November 2002)

\section{ABSTRACT}

Apyrases are enzymes that efficiently hydrolyze ATP and ADP and may operate both inside and outside the cell. Although apyrases are important to a variety of cellular mechanisms and uses in industry, there are no available apyrase-specific inhibitors. Colorimetric assays based on the Fiske-Subbarow method for measuring inorganic phosphate are able to detect the release of inorganic phosphate from ATP and other nucleotides. We found that this type of assay could be automated and used to screen for apyrase-inhibiting compounds by assaying for a reduction in released phosphate in the presence of potential inhibitors. The automation of this assay allowed for the successful screening of a commercially available compound library. Several low molecular weight compounds were identified that, when used at micromolar concentrations, effectively inhibited apyrase activity.

\section{INTRODUCTION}

Apyrases are enzymes that hydrolyze nucleoside diphosphates and triphosphates and are dependent on $\mathrm{Ca}^{2+}$ or $\mathrm{Mg}^{2+}$ for activity (13). The potato apyrase enzyme has a high catalytic activity with the ATPase $\mathrm{k}_{\text {cat }} \mathrm{K}_{\mathrm{M}}$ of approximately $10^{8} \mathrm{M}-1 \mathrm{~s}^{-1}(8)$. Apyrase enzymes have been implicated in many cellular functions, including golgi compartment protein glycosylation (6), depletion of parasite host cell ATP levels (15), purine salvage mechanisms (12), viral and cancer mechanisms $(4,13)$, and inhibition of platelet aggregation (1). Besides apyrase function in the cellular mechanisms mentioned above, potato apyrase specifically is an important industrial enzyme used in assays such as luciferase-based microbial contamination tests to clear samples of background ATP (16).

Despite the interest in apyrase function, there is a lack of useful inhibitors specific for apyrase. Typical inhibition of apyrase activity involves the use of ATP analogues such as ARL 67156 $(7,17)$ or a high concentration of sodium azide (11) or vanadate. However, these inhibitors may be impractical for use in some research or industrial applications and unsuitable as potential therapeutic agents. In fact, literature discussing apyrase is punctuated with comments regarding the lack of apyrase inhibitors $(2,14,18)$.

Colorimetric assays such as the Fiske-Subbarow and malachite green tests for the release of inorganic phosphate are widely used for measuring ATPase activity and other processes involving free phosphate $(3,10)$. Here we discuss an automated, high-throughput screen designed to obtain efficient and effective small-molecule inhibitors of apyrase from a library of compounds. This screen utilizes the Fiske-Subbarow assay and a commercially pre- 